\title{
Sequential Predation: A Multi-model Study
}

\author{
Jan D. van der LaAn $\dagger \|$, Ladislav Lhotka $\$$ and Pauline Hogeweg $§$
}

\begin{abstract}
$\uparrow \$$ Theoretical Biology Group, University of Utrecht, Padualaan 8, 3584CH Utrecht, The Netherlands and $\$$ Laboratory of Biomathematics, Institute of Entomology CAS, Branišovská 31, 37005 České Budějovice, Czech Republic
\end{abstract}

(Received on 6 May 1994, accepted in revised form on 19 October 1994)

\begin{abstract}
In many ecosystems food resources are available sequentially. The paper analyses a situation with two competing prey species both of which are consumed by a common predator species. Within a season the two prey species are available sequentially, although there may be an overlap. Three modelling methodologies are applied to this system: discrete dynamical systems (difference equations), individual-oriented event-driven simulations and cellular automata. The presence of the predator is shown to have a strong impact on the outcome of the prey species competition. The system of coexisting prey species changes to a system of founder-controlled competition. It appears that sequential predation can even have counterintuitive evolutionary consequences for the prey species. The species which appears later in the season will be more successful in its competition with the early species if it favours the predator; for example, by a high leaf palatability. Spatial structuring and topological issues are found to play a crucial role in both the ecological and evolutionary dynamics. The advantages of a multi-model approach are discussed.
\end{abstract}

\section{Introduction}

In the standard theory of interspecific competition, two categories of competition mechanisms are generally distinguished: see, for example, Begon et al. (1986) or Yodzis (1989): direct negative interactions among the competing species (interference competition), and concurrent utilization of a limiting resource (exploitation competition). There are of course other ways in which species can have indirect negative interactions. Holt $(1977,1984)$ uses the term apparent competition for another important situation when two competing species are adversely affected by a common predator. It is obvious that an increase in the density of prey species 1 will lead to an increase in predator abundance, which in turn will affect the density of prey species 2 .

The above-mentioned simple competition schemes assume straightforward causal relations. Furthermore, most of the analytical methods are based on static,

\| Author to whom correspondence should be addressed. equilibrium conditions (Tilman, 1982). In this paper we describe an ecosystem where the interspecific interactions are dynamic and cannot be a priori classified as positive or negative, at least not in an absolute sense. Short-term advantage may turn into massive losses in the longer term, and vice versa.

The situation we discuss here is a special case of apparent competition. Two competing prey species are consumed by a common predator, but their relative availability to the predator within the season is not synchronous - one of the prey species appears earlier than the other, although there may be an overlap. We call such an interaction scheme sequential predation. It is common in many plant-herbivore systems, for example in systems where insects feed on leaves or flowers. Shifted timing of budbreak or flowering period for different plant species results in the sequential availability of the resources to insects. Another example of sequential predation is the host plant alternation exhibited by many aphid species (Dixon, 1985). In this case, however, the preference for each host plant spans over several generations 
of aphids. A field study conducted by Futuyma \& Wasserman (1980) on oak forests indicates some counterintuitive phenomena connected with sequential predation.

Hence, our first aim is to show that seasonal succession of prey species can be an important factor that influences not only the predator but also, via feedback mechanisms, the competition among the prey species themselves. Furthermore, we will show that this can even have unexpected evolutionary consequences. The second point we want to make is related to spatial structuring. Localization of processes and interactions often results in interesting spatial structures which in turn can influence the dynamics on both the ecological and evolutionary time scale. Finally, the third intention of this paper is to compare three different modelling formalisms when applied to the same problem and demonstrate the advantages of this multi-model approach.

\section{The Ecosystem: Competing Tree Species and Insects}

Futuyma \& Wasserman (1980) performed a field study of a forest ecosystem consisting of two oak tree species, scarlet oak (Quercus coccinea) and white oak (Quercus alba), and foliage-feeding insects, namely larvae of the fall cankerworm (Alsophila pometaria). We took this system as a starting point for our model-based study of sequential predation.

Futuyma \& Wasserman (1980) describe this system as a sample where the plant species in minority-be it either scarlet or white oak - suffers greater herbivory. They explain this phenomenon by the fact that the insect larvae are dependent on both oak species due to the difference in the availability of palatable leaves. This is caused by a shift in timing of budbreak: scarlet oak breaks bud approximately 10 days before white oak. Larvae of the fall cankerworm hatch at about the time that scarlet oak comes into leaf. Since they cannot survive for more than two or three days without food (Mitter et al., 1979), they are entirely dependent on scarlet oak during early instars. However, they cannot complete their development on mature foliage, and when the leaves of scarlet oak are becoming unpalatable they migrate to the white oaks. So, at the end of their development the larvae depend on white oak.

The relation of larvae to the two tree species is, however, asymmetric. First, after hatching all larvae immediately have to find an early tree (scarlet oak) to feed on, whereas the transition to the late trees (white oak) is gradual and may span the period from budbreak of the late tree till the time when leaves of the early tree become unpalatable. Second, because there is only one generation of larvae during each season, the number of larvae that start feeding on early trees is necessarily greater than the number of those that eventually migrate to late trees. On the other hand, the late tree may suffer more extensively from the presence of larvae since the per capita consumption increases with their weight. The early tree may also benefit from the earlier start of photosynthetic assimilation.

Another important feature of this particular ecosystem is the fact that most processes are strictly local, including the dispersal of eggs, because the females of $A$. pometaria are wingless.

\section{Multi-model Approach}

We have developed three models using different formalisms:

- DDS-discrete dynamical system (difference equations). This methodology, along with ordinary differential equations, is used widely in ecosystem modelling. It is supported by extensive theory and a wide variety of analytical tools. We formulate a simple DDS model representing an abstract system with sequential predation, and use bifurcation analysis for studying the qualitative properties of equilibrium points with respect to changes in model parameters.

- Individual-oriented, event-driven simulation model. This is a relatively new methodology which is becoming popular in theoretical ecology (Hogeweg \& Hesper, 1979, 1990; Hogeweg, 1988a; Villa, 1992; Judson, 1994). Recent developments in hardware and software have made it feasible to define simulation systems by means of local interactions between individuals and to study the dynamics of these systems as a whole. In such models it is possible to define explicitly a sequence of events, and simulate processes even if they occur on different time scales. We use the Hobo individual-oriented modelling environment (Lhotka, 1994). Since models of this type are defined in terms of individuals and local processes, there is a clear biological interpretation for all model parameters.

- $C A$-cellular automaton. A cellular automaton is a collection of simple cells organized in a regular grid. Each individual cell can have several states which change according to a prescribed algorithm (next state function), typically involving the present state of the cell and of neighbouring cells. Thus the model is expressed in terms of local interactions between elements which represent 
space rather than individuals. It is well known that cellular automata with only a few states and a simple next state function can exhibit very interesting complex behaviour (Gardner, 1970; Wolfram, 1984). We use a multi-layer multiprocess stochastic cellular automaton, in which the state of each cell represents the presence of one individual (or clone). This type of cellular automaton is a very powerful tool for studying (eco)systems with spatial structure (Hogeweg, 1988b, 1989; Van der Laan \& Hogeweg, 1992; De Boer et al., 1993). We use the cellular automaton as a large-scale model of a forest ecosystem with sequential predation. As in the DDS model many properties of the organisms are lumped, for reasons of simplicity. It is possible to perform long simulation runs which are necessary for observing evolutionary trends. Unlike the DDS model, the CA model is defined on a fine-grained spatial structure.

In the main text we describe each of the models briefly before discussing its results. This description should be sufficient for a qualitative understanding of model behaviour. Appendices $\mathrm{A}-\mathrm{C}$ contain more detailed information about the implementation of the models.

\section{DDS Model: From Coexistence to Founder-controlled Competition}

The equations of our DDS model are derived from a simple Lotka-Volterra model with self-limitation for prey:

$$
\begin{aligned}
& X_{t+1}=X_{t}+a_{1} X_{t}-b_{1} X_{t}\left(X_{t}+g Y_{t}\right)-c_{1} X_{t} Z_{t} \\
& Y_{t+1}=Y_{t}+a_{2} Y_{t}-b_{2} Y_{t}\left(Y_{t}+g X_{t}\right)+c_{2} Y_{t} Z_{t} X_{t} \\
& Z_{t+1}=Z_{t}+e_{1} c_{1} X_{t} Z_{t}+e_{2} c_{2} Y_{t} Z_{t} X_{t}-d Z_{t} .
\end{aligned}
$$

In these equations $X$ and $Y$ are densities of early and late prey, respectively, and $Z$ is the density of the predator. We include sequentiality in this model by assuming that the number of predators that enter the second part of a season is proportional to the amount of early prey consumed. Therefore, predation on late prey is influenced by both predator density and early prey density. This results in the predation term $c_{2} Y Z X$ in the equations for $Y$ and $Z$. Further reasoning behind the particular form of the eqns (1) and the meaning and interpretation of parameters are presented in Appendix A.

\subsection{EQUILIBRIUM POINTS}

In the two-dimensional system of the prey species only, there is a stable equilibrium (provided $g<1)$. If predators are present $(Z>0)$, a different situation arises. After the third dimension $(Z)$ is added, this equilibrium with no predators may, for some parameter values, lose stability and a pair of new attracting equilibria emerge. One of these two new equilibria has a high density of the early prey species and a low density of the late prey species, whereas the other one has low density of early prey and high density of late prey. The nullclines of the system are shown in Fig. 1. In the same figure, trajectories to each of the two stable equilibria indicate their position. Therefore, depending on the initial situation one will obtain either a system dominated by the early species or a system dominated by the late species. This result clearly demonstrates the effect of the predator on the competition between the two prey species. In the absence of the predator the two prey species coexist, whereas in the presence of the predator the final outcome of the competition is dependent on the initial densities of the two prey species. This type of competition is called founder-controlled competition (Yodzis, 1989). However, in contrast to the classic examples of founder-controlled competition, the non-dominant species will not become extinct but will remain in the system at a low density.

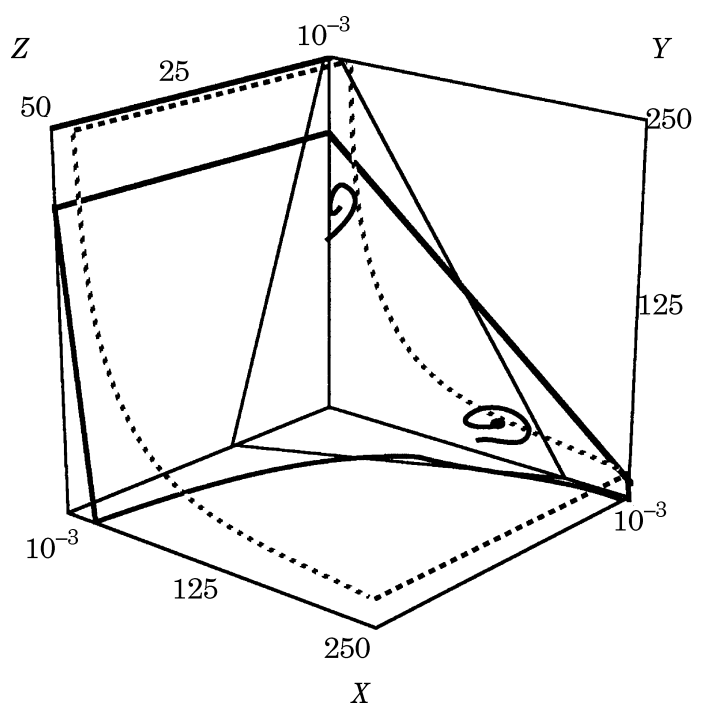

FIG. 1. State space of the DDS model, showing nullclines and equilibria. Flat plane is nullcline of early prey $(X)$, curved plane with bold lines is nullcline of late prey $(Y)$ and curved plane with dashed lines is nullcline of predator $(Z)$. Parameters: $a_{1}=a_{2}=1$; $b_{1}=b_{2}=0.005 ; g=0.75 ; d=1 ; c_{1}=0.05 ; c_{2}=0.00075 ; e_{1}=0.00005$; $e_{2}=0.3$. 


\subsection{BIFURCATION ANALYSIS}

The asymmetry in the two predator-prey relations is expressed explicitly in eqns (1): specific rate of predation on early prey depends only on predator density, whereas on late prey it depends on both predator and early prey density. Consequently, predator density has a different effect on each of the two prey species. An increase in predator density will always result in a decrease in the density of the early prey. It is more complicated to see the effect of an increase in predator density on late prey, since the increase in predators will to some extent be compensated by the decrease in early prey. We studied this effect by performing a bifurcation analysis of the system using the parameters, $e_{1}$ and $e_{2}$ (which are the parameters of the numerical response of the predator). Bifurcation diagrams are presented in Fig. 2. Figure 2(a) shows that the position of the equilibria does not change significantly with changing $e_{1}$. Figure 2(b) however shows a more interesting result: for small $e_{2}$ there is a stable (symmetrical) equilibrium with both prey species present, but with $Z=0$. At $e_{2}=0.10$ there is a transcritical bifurcation: the symmetrical equilibrium becomes unstable, whereas one asymmetrical equilibrium emerges with early prey dominant. If $e_{2}$ is further increased the system goes through a saddle-node bifurcation at $e_{2}=0.25$ : a second asymmetrical equilibrium is born, with late prey dominant. The basin of attraction of this new equilibrium becomes larger if $e_{2}$ is further increased. Therefore, it is beneficial for late prey if $e_{2}$ is large. This result means that a mutant of late prey with higher nutritional value for the predator will perform better in competition with early prey than will a wild type.

However, if such a mutant were to appear in a wild-type population of late prey it would never be able to increase its density substantially. Once the system is trapped in the equilibrium with early trees dominant, an increase in $e_{2}$ will not cause the system to move to the other equilibrium. Moreover, even if it were possible to make this move to the equilibrium with dominance of late trees, then both the mutant and the wild-type late tree would profit from this change. Thus if mutants were to appear in the system, their frequency (relative to wild type) would not increase.

\section{The Individual-oriented Model}

The individual-oriented Hobo model of sequential predation is closely related to the ecosystem described by Futuyma \& Wasserman (1980). However, we do not treat our simulations exclusively in the context of the particular situation. Instead, we adopt the following
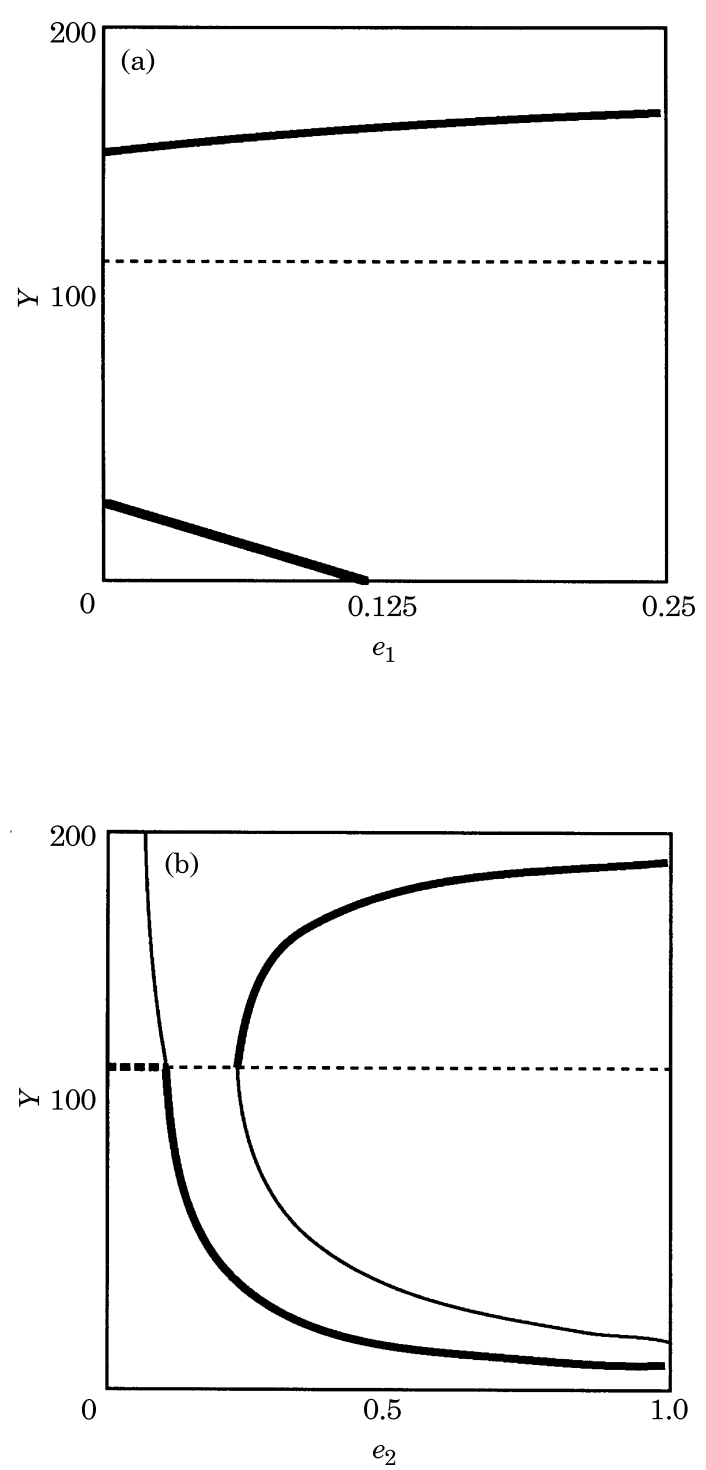

FIG. 2. Stability of equilibria of the DDS model as a function of $e_{1}$ (a) and $e_{2}(\mathrm{~b})$. Dashed straight line is the continuation of the symmetric $(X=Y ; Z=0)$ equilibrium; other lines are the continuation of the 3-D equilibria. At the bold line section equilibria are stable, unstable equilibrium otherwise. Parameters: $a_{1}=a_{2}=1$; $b_{1}=b_{2}=0.005 ; g=0.75 ; d=1 ; c_{1}=0.05 ; c_{2}=0.00075 ; e_{1}=0.00005 ;$ $e_{2}=0.3$.

qualitative assumptions about our "imaginary" system of two species of trees and insect larvae that feed on the leaves of both species:

- Both tree species have localized reproduction, i.e. the spread of seedlings is limited to the neighbourhood of the parent tree.

- A priori competitive advantage of the early trees results in an increased production of viable seedlings and decreased mortality.

- Ten age classes of trees are distinguished. A new tree that had just colonized an empty patch is 
assigned age 1. Each season this number is increased by 1 , but only up to 10 . After that the age does not increase and the tree is considered mature. Several parameters depend on tree age: number of leaves, number of viable seedlings, and mortality.

- A tree dies with a certain probability which is proportional to the defoliation of the tree and inversely proportional to the tree's age.

- Whenever a tree dies, its patch is taken over by a new young tree. The species of this invader is determined probabilistically according to which species is more abundant in the "seedling bank" of the empty patch.

- The larval development has to finish within 32 days after hatching. A larva can successfully pupate only if its weight is above a certain threshold.

- However, the metamorphosis to pupa can take place sooner if a larva reaches another-higherthreshold.

- Additionally, we assume the larvae develop through four instars. Thus, every 8 days after hatching all larvae in the system are checked for weight. Those that are below a prescribed value are immediately killed.

- Food demand of larvae increases exponentially during development and the leaf palatability, hence the total utilizable food resources, decrease. Therefore, near the end of development it is considerably harder to find enough food than just after hatching.

- The larvae migrate to neighbouring trees either via overlapping branches, which is a common and relatively safe way, or by leaving the host tree and moving on the ground to another one, this move being connected with an increased risk of predation. We assume the latter dangerous way is used only by larvae leaving a tree with no foliage or with unpalatable foliage.

- The larvae search for better food resources by means of trial-and-error only.

- We assume the adult insects have a 1:1 sex ratio and the number of eggs produced by each female is proportional to the weight reached at the end of the larval stage.

- The standard variant of the model assumes wingless females, which implies local reproduction of the insects. A female is assumed to lay eggs on the tree where it completed its larval development, or on one of its immediate neighbours.

The temporal scale consists of discrete seasons, each of them being 100 time units (days) long, i.e. seasons start at times which are multiples of 100. Every season is punctuated by the following events:

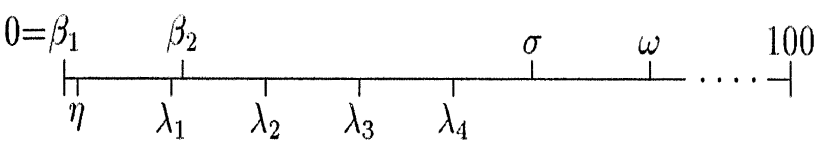

The events related to trees are $\beta_{1}=0$ and $\beta_{2}=10$ : the time of budbreak of early and late trees, respectively; $\sigma=40$ : here both species produce seedlings; and $\omega=50$ : end of the season when the new composition of the model forest is determined. The larval life cycle contains the events $\eta=1$ : time of hatching, and $\lambda_{i}=8 i+1$, which is the deadline for finishing the $i$-th instar, for $i=1,2,3,4$. Note that after $\lambda_{4}$ it is only the sequence of further events which is important and the exact timing of $\sigma$ and $\omega$, and the length of the season are irrelevant.

The Hobo model is built on a regular lattice of square patches. Every patch is regarded as a stand for a single tree and retains a specific set of parameter values. Trees are thus defined as properties of patches - in the following text we effectively identify trees with their patches.

A detailed description of how the Hobo model is implemented is given in Appendix B.

We use two sizes of simulated forest: $10 \times 10$ and $32 \times 32$ patches. In order to generate representative seedling banks in all patches, we let all simulations first run 100 seasons without larvae. Thus, the interesting part of each simulation begins only at time 10000 and is 25 seasons long (until 12500). Initial distribution of larvae (i.e. those hatching at time 10001) is random, each patch being given 0-20 larvae (with uniform probability distribution).

The model and the Hobo simulation system itself are written in the Smalltalk-80 language (ParcPlace Systems, 1992). The simulations were carried out on a UNIX workstation (HP 9000/720). The longest runs on the $32 \times 32$ scale took about 40 hours to complete.

\subsection{BASIC MECHANISMS}

To understand the major forces that drive model dynamics, we first use a simple spatial configuration on the smaller scale of $10 \times 10$ patches. We start with a forest composed of early trees except for a $2 \times 2$ rectangle in the centre which contains four late trees.

Simulation results are visualized in Figs 3 and 4. Figure 3 shows the time dependence of cumulative numbers of insect eggs - we will use this quantity throughout as a good interseasonal measure of population density. We observe pronounced oscil- 


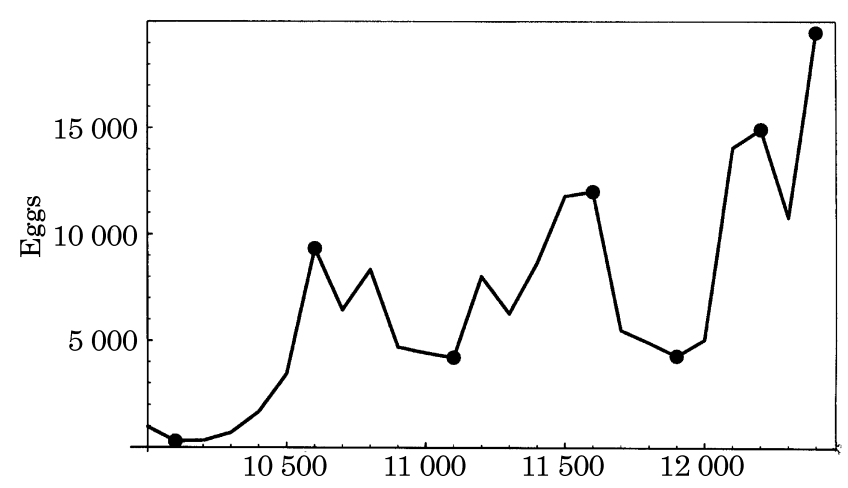

FIG. 3. Time plot of the number of eggs in the small-scale $(10 \times 10)$ simulation with an initial minority of late tree. Dots indicate the seasons when the snapshots in Fig. 4 were taken.

lations but with an overall increase. This behaviour can be explained using Fig. 4 which shows the spatial distribution of four quantities recorded during critical seasons before extremes in egg numbers were achieved, as indicated by dots on the graph in Fig. 3. In the "tree" column we see how the initially small island of late trees (black squares) is steadily expanding. The snapshots of larvae distribution (second column) are taken at time 9 of each season (end of the first instar). Naturally, almost all larvae are found on early trees since it is just before the late trees break bud. The third column, "eggs", shows an almost complementary layout - most eggs are laid on late trees. Finally, the "foliage" column shows the distribution of foliage, which reflects both the age of trees and level of defoliation during the season.

In the first season (denoted as 100) most of the larvae were not able to find their way to the late trees and died. Eggs were laid on two trees only. During the following five seasons the larval population grew steadily. In the second row of Fig. 4 we see relatively high densities of larvae and eggs in the central part. The larvae that hatch in large numbers on late trees have to migrate immediately to an early tree. Hence, early trees in the neighbourhood of the island of late trees are intensively defoliated, some of them die, and so season after season the larvae have to travel further and further to find enough food on early trees. The larger this dispersal, the fewer larvae find their way back to the late trees towards the end of the season. All these factors increase the mortality of larvae. At some point, when the defoliated ring is sufficiently large, most larvae cannot be successful in this back-and-forth migration and population density decreases. In the column on the far right of season 105 we can clearly see the defoliated "firewall" around the island of late trees.

When the density of larvae drops, the defoliated gap can recover again. Due to the asymmetry in predation pressure on the two tree species, the late trees have good chances of expanding into the free space. In this way the gap is partly closed, the task of larvae becomes easier and their population grows again. This process is repeated cyclically and leads to the oscillations observed in Fig. 3.

From the above analysis we can also deduce that the most important part of the space is the boundary between the areas occupied by different tree species. The length of this boundary increases during the simulation due to the expansion of late trees, and accordingly there is an increase in the maximum population size that can be supported in a particular configuration (cf. Fig. 3).

Is it true here that the species in the minority suffers greater herbivory, by analogy with the conclusion of Futuyma \& Wasserman (1980)? Of course, such a comparison depends strongly on which sites are selected for taking samples. As long as the area occupied by late trees is small, most of the early trees are unaffected by the presence of larvae, for these have to stay close to the late trees. If we then take samples of both trees at random positions, as Futuyma \& Wasserman (1980) probably did, we may very well find the sampled early trees to be less damaged on average than the sampled late trees, even though the minority (late) species has a clear competitive advantage just as in our simulation.

However, when the early tree is in the minority, it really suffers more, by all measures, than the late tree. To see this, we repeated the same simulation experiment as above, but interchanged the roles of the tree species in the initial configuration: that is, we started with a $2 \times 2$ block of early trees in the centre and late trees elsewhere. The story was rather trivial this time. A growing population of larvae was established in the centre again where it caused gradual defoliation. In this case, however, all the early trees were on the boundary of the late trees and once the early trees had been defoliated the system broke down immediately and all larvae died.

\subsection{EFFECTS OF SPACE CONFIGURATION}

In order to test the effects of spatial configuration on the larger scale $(32 \times 32$ cells), we used three different arrangements of the tree species in space: random configuration (type A), simulated forest (B), and complete separation(C). At time 10000, when the larvae are introduced, the three configurations are as in Figs 5(a)-(c).

First we ran three simulations, $1 \mathrm{~A}, 1 \mathrm{~B}$ and $1 \mathrm{C}$, all using the standard parameter setting described in Appendix B. Figures 5(d)-(f) show the status of 
the forest at the end of each simulation, i.e. after 25 seasons. Quantitative results are presented in Fig. 6. We observe a monotonous decrease in the number of early trees in all three cases, yet at different rates [Fig. 6(a)]. Egg counts depend sensitively on the configuration, as shown in Fig. 6(b).

With configuration $\mathrm{C}$ a relatively small population of larvae was established near the borderline between early and late forests and this population remained relatively stable during the entire simulation. The borderline itself moved slowly to the left in the same way as we saw before in the small-scale simulation. The size of the larval population is not affected by this expansion of late trees as long as the borderline does not come close to the left edge of the forest.

Conversely, the initial increase in the abundance

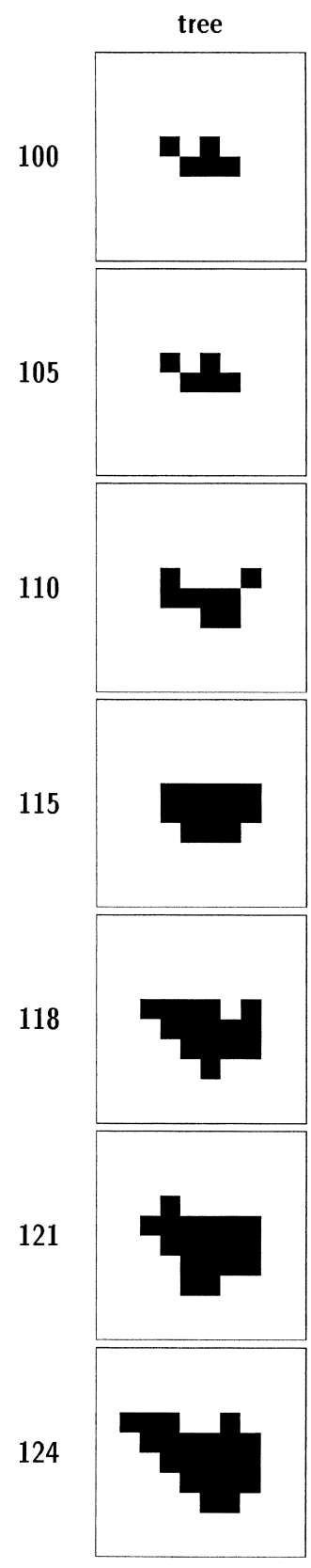

0
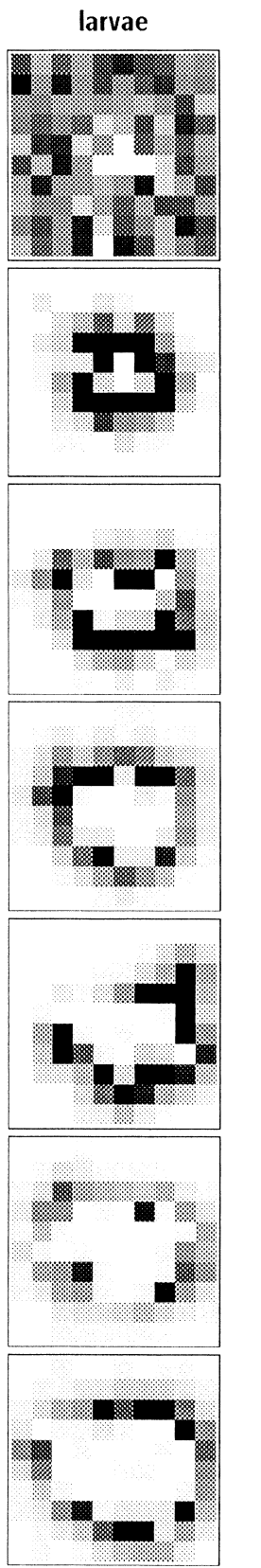

9
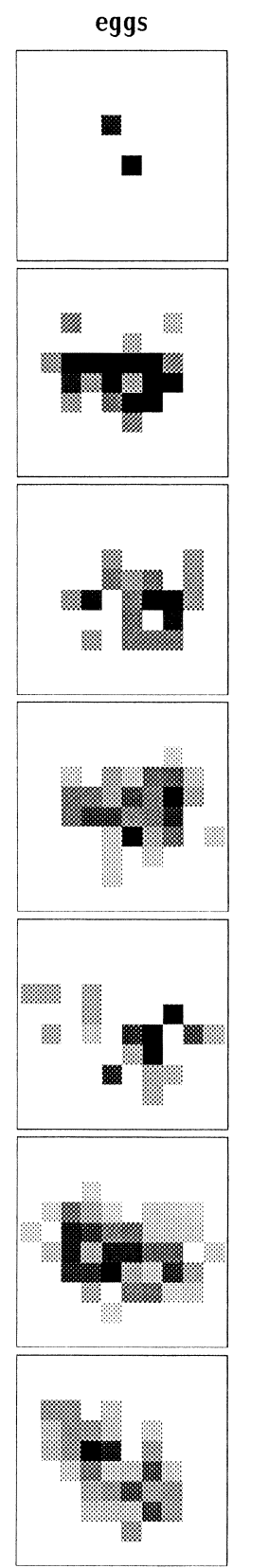

40
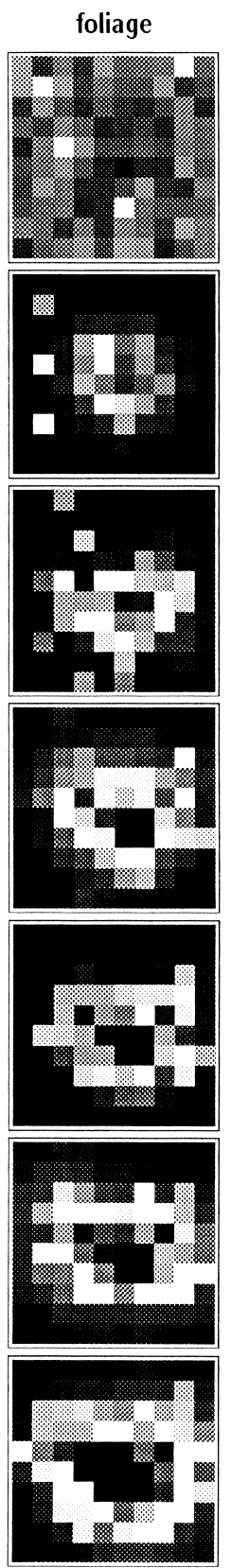

40

FIG. 4. Density plots of the distribution of trees (early= white, late=black), abundance of larvae, number of eggs and foliage, for seven seasons of the small-scale $(10 \times 10)$ simulation with an initial minority of late trees. Numbers on the left are the sequential numbers of the season, and numbers at the bottom give the time within the season when the snapshots were taken. The shades of grey are comparable throughout each column. 
(a)

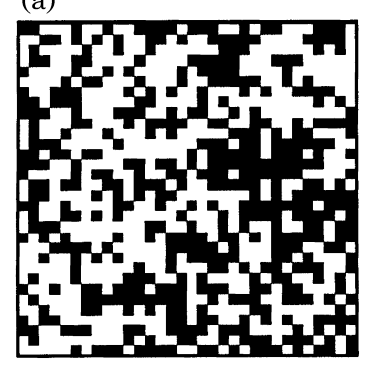

(b)

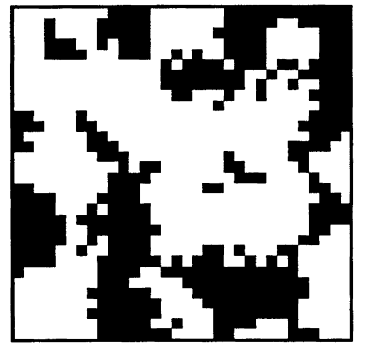

(c)

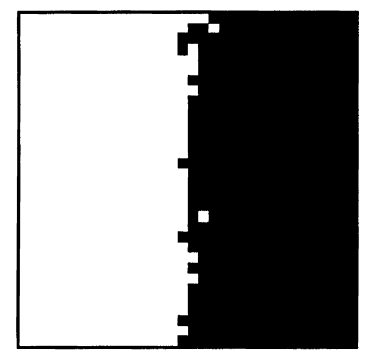

FIG. 5. Distribution of trees (white $=$ early, black $=$ late) for simulations 1A (a, d) 1B (b, e) and 1C (c, f). Figures (a), (b) and (c) show the situation just before the larvae have been introduced (time $=10000$ ) and (d), (e) and (f) show the final situation (time $=12500$ ). See text for more description.

of larvae is very fast in a random configuration (simulation 1A), which leads to a rapid defoliation of all early trees [see Fig. 6(c)] and after another small oscillation the larvae die out. This is caused by the fact that almost all trees have neighbours of the other species; hence the boundary-based competition mechanism is very intensive.

As expected, the dynamics of the simulated forest (simulation 1B) is somewhere in between the extreme cases $1 \mathrm{~A}$ and $1 \mathrm{C}$. The same competition mechanism works here - the expansion of late trees proceeds along the entire boundary of the areas of early and late trees, cf. Fig. 5(b), (e).

Therefore, in summary we can say that the most crucial topological property of this sequential predation system is the character of the interface between the areas occupied by early and late trees, respectively - the more interwoven are the two areas, the faster is the competition process which leads here to an exclusion of the early trees.

\subsection{LARVAE AS BIOLOGICAL WARFARE}

The outcome of competition between the two tree species is largely determined by the presence of larvae which favours the late tree. An increase in the density of larvae should thus amplify the competition processes. We will now show that due to this fact it is advantageous for late trees to "support" the larvae even if the late trees themselves suffer from an increased herbivory.

To this end, we set up another simulation, 2B, configured as a simulated forest. The difference between this simulation and simulation $1 \mathrm{~B}$ is the use of a different leaf palatability function for late tree. Both tree species have leaf palatability equal to 1 for leaf maturation $\tau$ (time since budbreak) or less than 8 days. For $\tau \geqslant 8$ we use the palatability function $q^{\prime}(\tau):=(48-\tau) / 40$ for late trees, while for early trees the standard function $q(\tau):=(28-\tau) / 20$ is used.
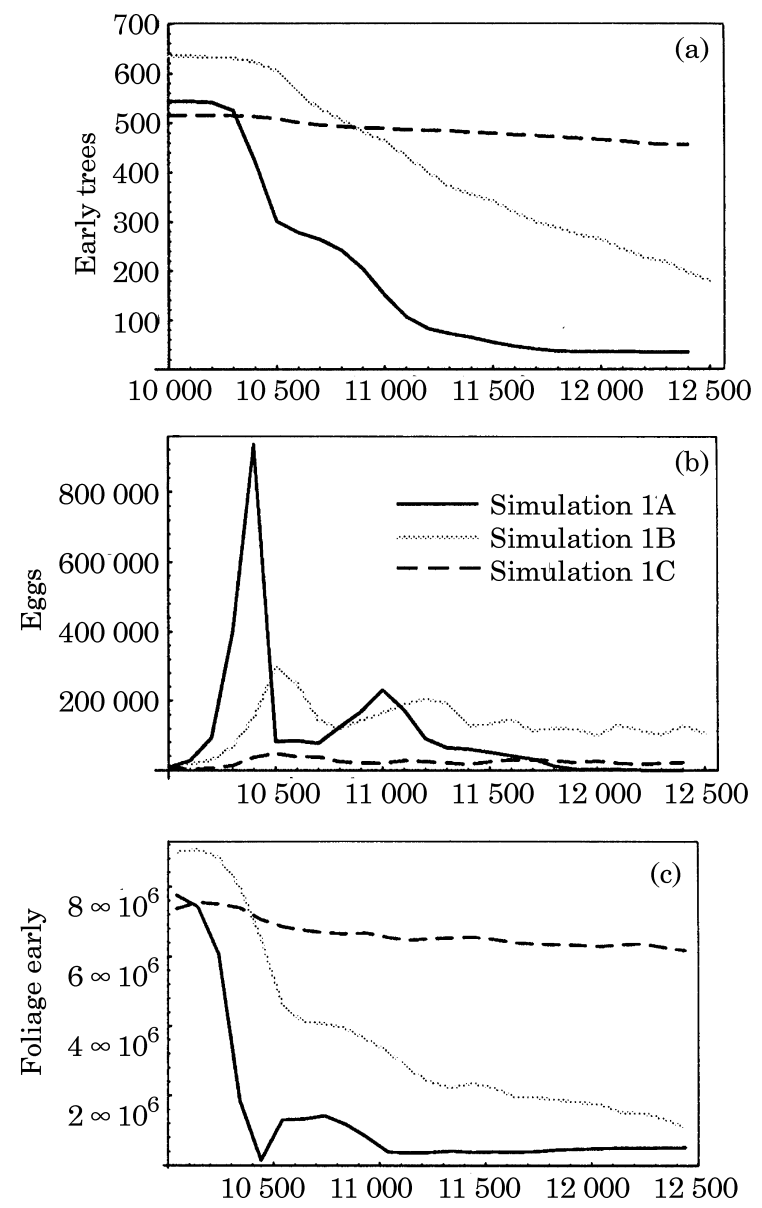

FIG. 6. Time plot of the number of early trees (a), number of eggs (b) and foliage on early trees (c) in simulations 1A (full black line), 1B (grey line) and 1C (dashed line). 


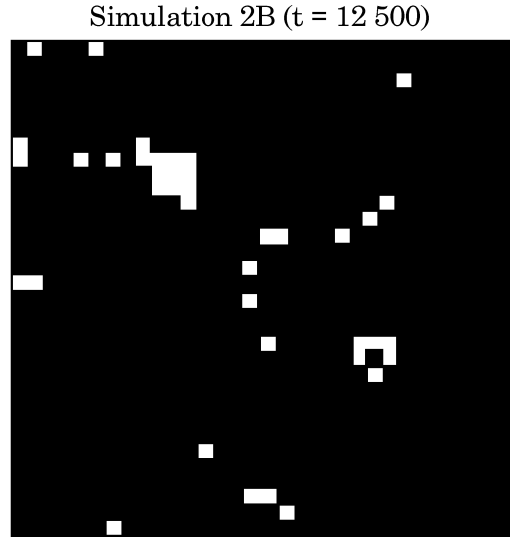

FIG. 7. Final distribution of trees in simulation $2 \mathrm{~B}$ (early $=$ white, late $=$ black).

This modification makes leaves of late tree relatively more palatable, especially towards the end of the season.

Figure 7 shows the status of the forest at the end of the simulation. When compared to the final picture of the simulation 1B with default (faster decreasing) leaf palatability function [Fig. 5(e)], we see considerably fewer early trees here. The dynamics of the number of eggs is shown in Fig. 8(a), for the two simulations 1B and $2 \mathrm{~B}$. In the initial phase the larval population grew much faster in $2 \mathrm{~B}$. Later the two curves are not significantly different, but the process of exclusion of
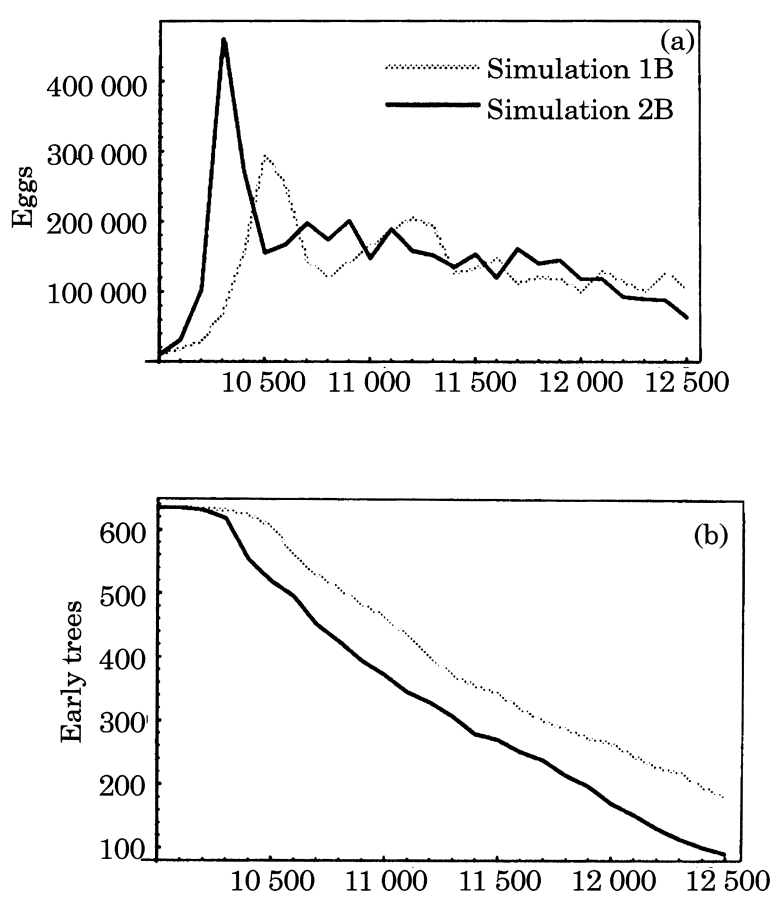

FIG. 8. Time plot of the number of eggs (a) and number of early trees (b) in simulation 2B (black line), compared to simulation 1B (grey line). early trees in $2 \mathrm{~B}$ is about four seasons ahead of $1 \mathrm{~B}$, as we can see from Fig. 8(b).

These results thus demonstrate that a late tree can indeed increase its competitive advantage by providing more valuable food for the larvae. The damage to a late tree caused by the increased abundance of larvae is more than outweighed by the high losses of early trees in the initial phase of each season.

\subsection{DISPERSED VERSUS LOCAL REPRODUCTION OF THE INSECTS}

We have already seen that the larvae have to stay close to the boundary between early and late trees because of the back-and-forth migration. The localized character of insect reproduction (wingless females) is in accord with this requirement in that the eggs are also laid close to the boundary. It is natural to ask what would happen if the insect females had wings and eggs were thus dispersed over the space. This question was investigated for the same three spatial configurations as studied before, using a different algorithm for the distribution of eggs: first the eggs produced by all females are accumulated and then dispersed evenly over the entire space.

With configuration $\mathrm{C}$ (complete separation of the tree species) the outcome is the easiest to predict. Because most eggs are laid far from the borderline between the early and late forests, the viable part of the larval population (located near the borderline) is strongly diluted in each season. Indeed, population density of larvae in corresponding simulation 3C rapidly decreased and after four seasons there were no larvae left.

In a random configuration (simulation $3 \mathrm{~A}$ ) virtually all patches are on the boundary of the early and late forests. Therefore, there should be no topological difficulties for larvae such as existed in the preceding case. What happened is shown in Fig. 9. The larvae did not survive much longer than in the previous situation. This time, however, the population density underwent first an explosive growth and attained much higher value at the first peak than in the case of local reproduction [Fig. 9(a)]. Dispersed reproduction permitted a more efficient utilization of available resources, but resulted in a global and absolute defoliation of early trees [see Fig. 9(b)]. After losing this critical resource, the larvae died out immediately.

It is not clear a priori which of the two factors will dominate in simulation 3B (simulated forest). However, Fig. 9(c) and (d) show dynamics that is similar to but slower than the dynamics as in the case of random configuration. Apparently, the topological constraints were too weak to prevent the initial defoliation. 

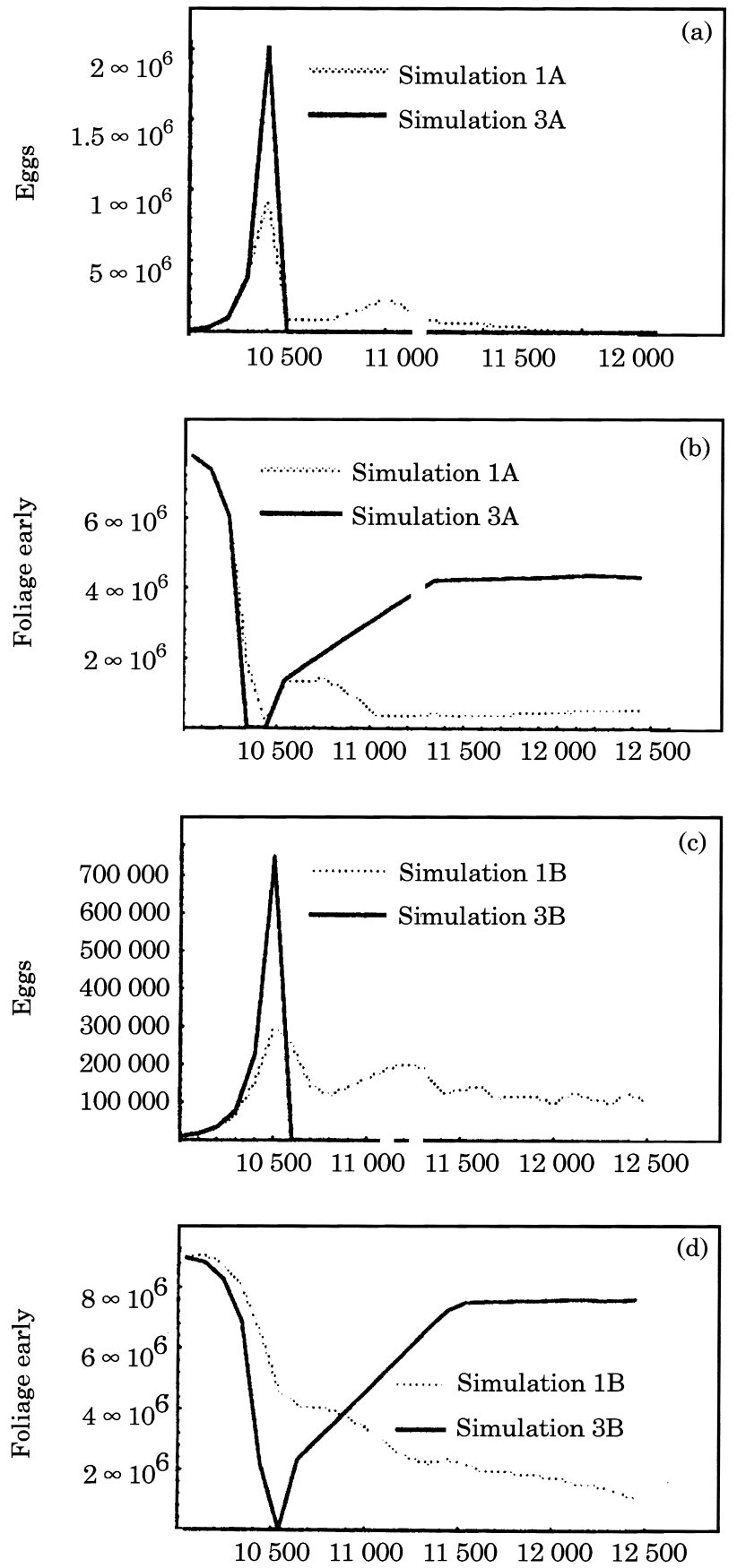

FIG. 9. Time plot of the number of eggs (a, c) and foliage (b, d) on early trees. Figures (a) and (b) show these quantities for simulation 3A (black line) and simulation 1A (grey line). In figures (c) and (d) a comparison has been made between simulation 3B (black line) and simulation 1B (grey line). The values in each season were taken at time 40 .

Despite the fact that qualitatively different mechanisms govern these three simulations, we found dispersed reproduction to be a destabilizing factor which in all cases leads to the extinction of larvae.

\section{CA Model: Evolutionary Consequences of Sequential Predation}

The cellular automaton model of sequential predation is defined in terms of simple local variables and interactions in a spatial ecosystem. Every cell represents a patch of space with qualitative states (present or absent) for trees and insects. The different processes which act on the variables are modelled as a sequence of cellular automaton rules. For a detailed description of the model we refer to Appendix C.

The rectangular space of the simulated forest consists of $240 \times 120$ patches. The tree dynamics is characterized by local processes: the colonization of empty patches is dependent on the species composition of neighbouring patches. This localized reproduction implies competition for space both within and between tree species. The mortality of trees is effected by three different factors:

- A tree can die in a particular season with a certain probability $M_{g}$. We will call this global mortality, since it is independent of the local situation.

- A tree surrounded by other trees of its own species has an extra probability of dying $M_{l}$. This extra density-dependent mortality, which we call local mortality, is added to the model in order to take the effect of intraspecific competition into account. Without this local mortality intraspecific and interspecific competition of the trees would be equal ( $g=1$ in the DDS model).

- Insect defoliation also affects the probability that a tree will die $\left(M_{i}\right)$. Since the insects increase in weight during the season, mortality due to the presence of insects is assumed to be higher for trees of the late species than for trees of the early species: $M_{i}(2)>M_{i}(1)$.

With respect to insect dynamics we will assume that the sequential predation is obligatory. This means that during the first half of the season insects can only survive on early trees, whereas in the second half they can only survive on late trees. When switching from early to late tree, insects migrate by a passive diffusion process, which can take them to a distance of at most five patches. At the end of the season insects reproduce locally, which means that they can colonize some of the surrounding trees in an area which consists of a square of $5 \times 5$ patches (with the source tree in the middle). The proportion of trees of this area $\left(P_{r}\right)$ which they actually will colonize is the key parameter to manipulate. This parameter can be regarded as equivalent to the parameter $e_{2}$ in the DDS model. A nutritious tree of the late species (or a late tree with less 
tannin in its leaves) will contribute to a higher reproduction of the insects that live on that tree. In the model this would mean a higher value for $P_{r}$.

New insects can originate by reproduction or as a result of global influx. Global influx has been added to the model in order to guarantee the presence of insects during the whole simulation.

\subsection{DOMINATED FORESTS AND THE EFFECT OF INSECTS}

We start our simulations with a forest consisting of $50 \%$ early and $50 \%$ late trees randomly distributed. An initial period without insects results in a stable mixed

(a)

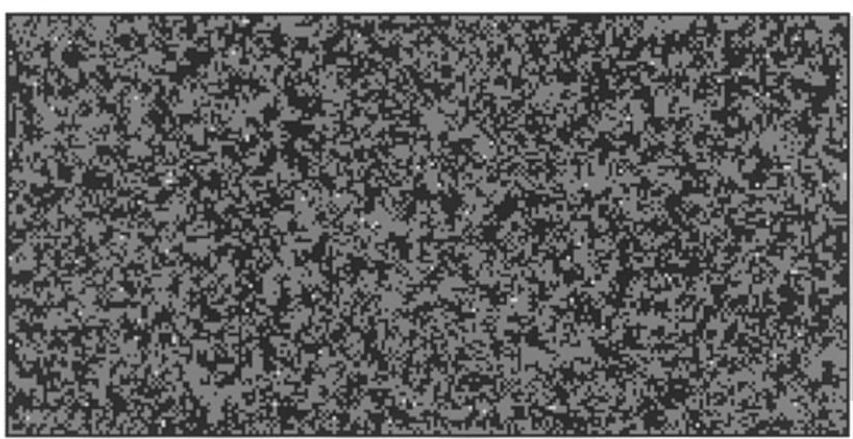

(b)

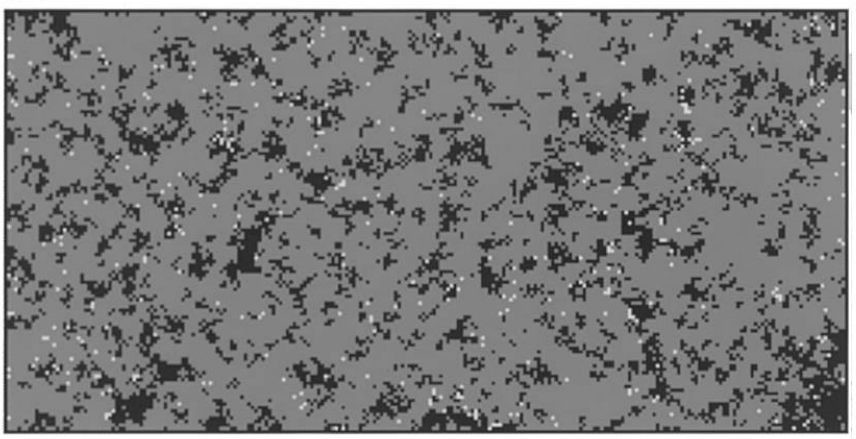

(c)

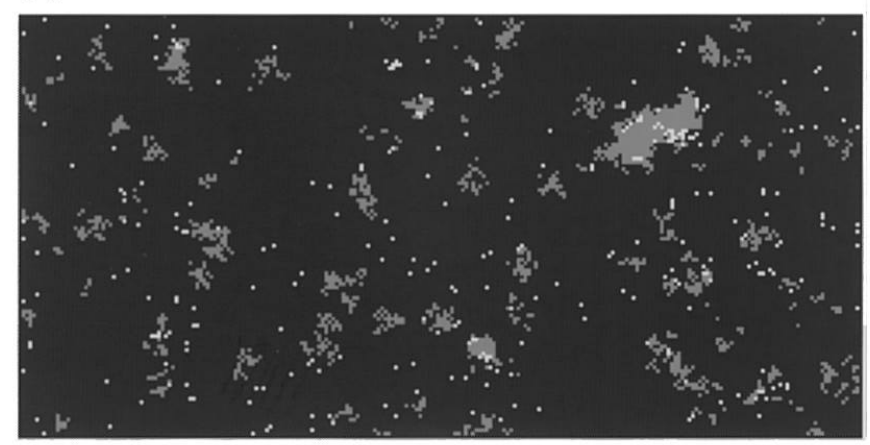

FIG. 10. Distribution of trees (white=empty patch; light grey $=$ early; dark grey $=$ late). Figure $(a)$ shows the situation without insects $(t=0)$, figures (b) and (c) show a forest at $t=1000$ with insects, having low reproduction, $P_{r}=0.4$ (b) or high reproduction, $P_{r}=0.7$ (c) Parameters: $\quad M_{g}(1)=M_{g}(2)=0.03 ; \quad M_{l}(1)=M_{l}(2)=0.05$; $M_{i}(1)=0.35 ; M_{i}(2)=1.0 ; I=0.001$. (a)

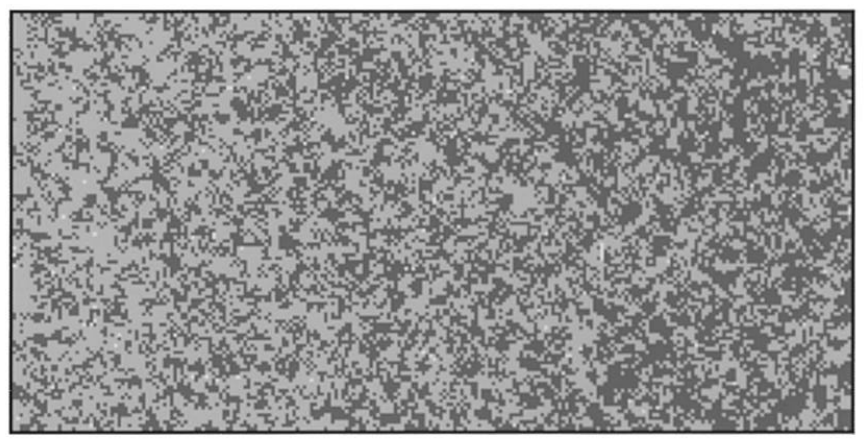

(b)

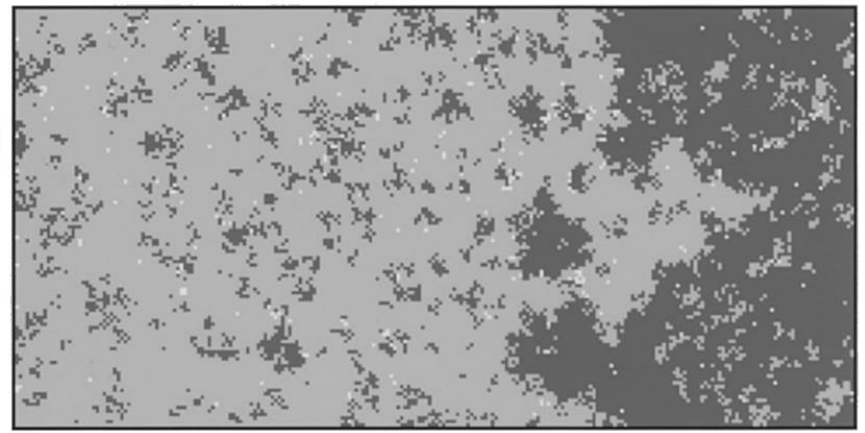

(c)

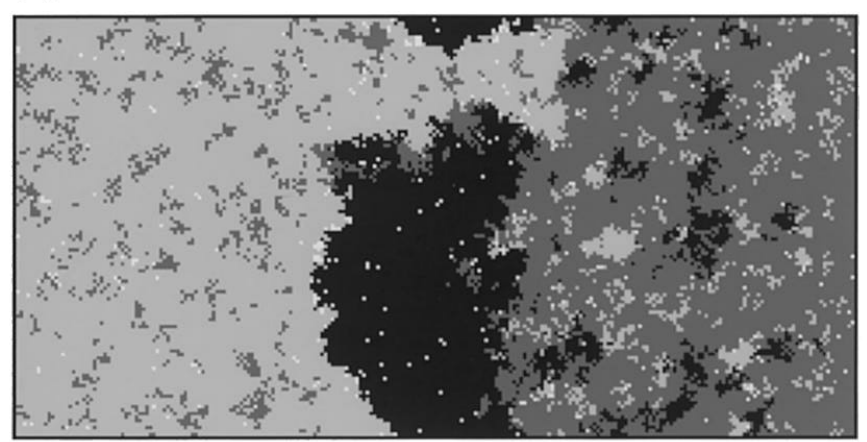

FIG. 11. Distribution of trees on a gradient with localized insect reproduction (white =empty patch; light grey=early; dark grey $=$ late; black = mutant). Figure (a) shows the situation without insects $(t=0),(\mathrm{b})$ shows the situation with insects at $t=1000$, and (c) shows the situation at $t=2500$ (after the introduction of mutants of the late tree at $t=1000)$. Parameters: $M_{g}(1)=$ gradient from 0.02 to $0.04 ; \quad M_{g}(2)=M_{g}(m)=0.03 ; \quad M_{l}(1)=M_{l}(2)=M_{l}(m)=0.05 ;$ $M_{i}(1)=0.35 ; \quad M_{i}(2)=M_{i}(m)=1.0 ; \quad P_{r}(2)=0.5 ; \quad P_{r}(m)=0.95 ;$ $I=0.001$.

forest with a small-scale patchiness of tree distribution, due to the localized reproduction [Fig. 10(a)]. We then introduce insects in $0.1 \%$ of the trees, randomly distributed over the forest, and simulate the forest for 1000 years. We do this for two different values of $P_{r}$, the reproduction of insects. The positive effect of a high insect reproduction on trees of the late species is evident. Low $P_{r}$ results in a forest dominated by early trees [Fig. 10(b)], whereas high reproduction results in a forest dominated by late trees [Fig. 10(c)]. 


\subsection{EVOLUTIONARY CONSEQUENCES}

The results of all three models clearly demonstrate that high insect density means a competitive advantage for the late tree. We further investigate this interesting phenomenon by introducing a rare mutant of the late tree with properties that lead to higher reproduction of the insects that feed on it. In Section 4 we argued that once the system is in equilibrium, introduction of such a mutant would never change the outcome. Here, we study the fate of a mutant if local interactions are present in the model. We will do this in a setting slightly different from the one used in Section 6.1. By adding one extra property to our model forest, i.e. by assuming a gradient of a factor influencing the global mortality of the early tree $\left(M_{g}(1)\right)$, we can see gradual changes in tree composition in one cellular automaton. Near the left edge of the cellular automaton early trees have a lower global mortality than late trees, and vice versa near the right edge. Figure 11(a) shows a forest on such a gradient. After the introduction of the insects the pattern of gradual change in tree abundances changes to a pattern of two distinct regions [Fig. 11(b)]. In the left part of the forest trees of the early species are dominant, whereas in the right part of the forest the late tree is the dominant species. The qualitative properties of the spatial pattern, i.e. two different types of forest divided by a sharp boundary, have been observed by Futuyma \& Wasserman (1980) in their study of oak forests in Suffolk County.

The above result of our CA model can be explained by further analysis of the DDS model. Figure 12 shows a bifurcation plot of the DDS model using the

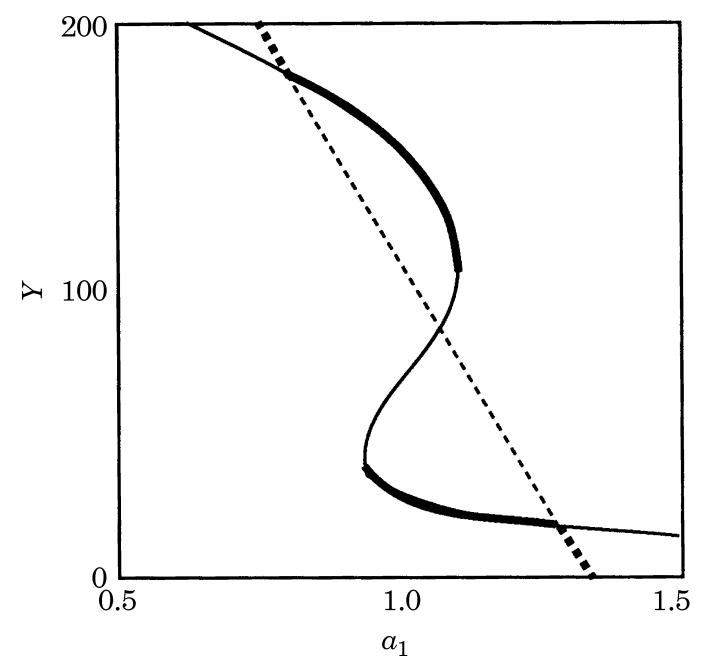

FIG. 12. Stability of equilibria of the DDS model as a function of $a_{1}$. The dashed straight line is the continuation of the symmetric ( $X=Y ; Z=0$ ) equilibrium, the curved line is the continuation of the 3 -D equilibria. At the bold line section equilibria are stable, unstable equilibrium otherwise. Parameters: $a_{2}=1 ; b_{1}=b_{2}=0.005 ; g=0.75$; $d=1 ; c_{1}=0.05 ; c_{2}=0.00075 ; e_{1}=0.00005 ; e_{2}=0.3$. (a)

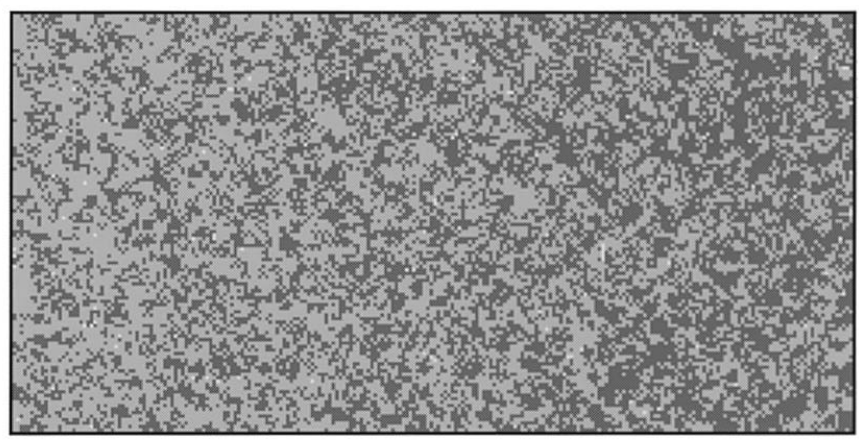

(b)

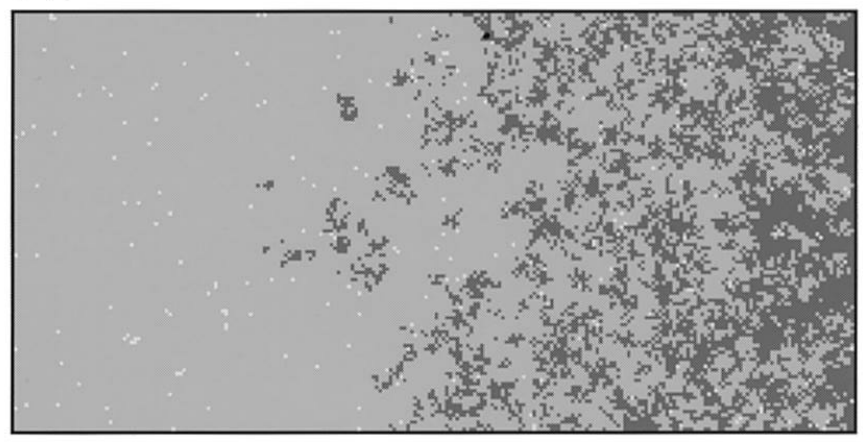

(c)

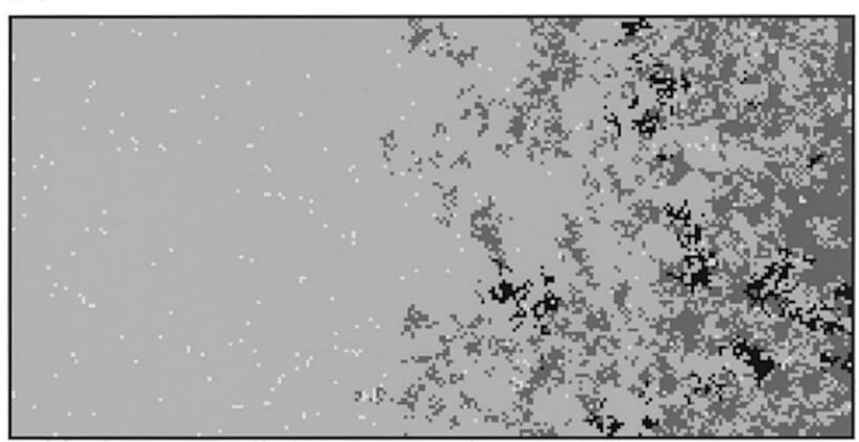

FIG. 13. Distribution of trees on a gradient with dispersed insect reproduction (white $=$ empty patch; light grey=early; dark grey $=$ late; black = mutant). Figure (a) shows the situation without insects $(t=0),(\mathrm{b})$ shows the situation with insects at $t=1000$, and (c) shows the situation at $t=2500$ (after the introduction of mutants of the late tree at $t=1000)$. Parameters: $M_{g}(1)=$ gradient from 0.02 to 0.04; $\quad M_{g}(2)=M_{g}(m)=0.03 ; \quad M_{l}(1)=M_{l}(2)=M_{l}(m)=0.05 ;$ $M_{i}(1)=0.35 ; \quad M_{i}(2)=M_{i}(m)=1.0 ; \quad P_{r}(2)=0.5 ; \quad P_{r}(m)=0.95 ;$ $I=0.001$.

parameter $a_{1}$ as a bifurcation parameter. With $Z=0$ (no insects) the equilibrium (dashed line) changes gradually from a dominant late forest to a dominant early forest with increasing $a_{1}$ (decreasing mortality). With $Z>0$ the continuation of a $3-\mathrm{D}$ equilibrium results in a catastrophe fold with two stable equilibria for either a late-tree dominated system or for an early-tree dominated system. Starting without insects (i.e. starting on the dashed line) the equilibrium with the late tree dominant will be attained at low 
$a_{1}$, whereas the system will settle into the other equilibrium at high $a_{1}$.

Into this system of "dominated" forest regions, i.e. in the system of Fig. 11(b), we introduce a mutant of the late tree species. The tree dynamics of mutant and wild-type tree are exactly the same. The only difference between mutant and wild-type trees is their effect upon the insects: insects which feed on mutant trees produce more offspring $\left(P_{r}(m)>P_{r}(2)\right)$. After a small fraction of the late trees is changed into a mutant tree the spatial structure of the forest changes significantly [Fig. 11(c)]. The mutant becomes the dominant tree in a large region of the forest, which used to be dominated by the early species. Therefore, it is possible for a mutant to invade the spatial system. In the region initially dominated by late trees mutants can survive, but they are not able to replace the wild type.

\subsection{DISPERSED VERSUS LOCAL REPRODUCTION OF INSECTS}

The mutant in the previous simulation is more successful than in the non-spatial DDS model because the mutant benefits relatively more from the positive effect of more insects than does the wild-type late tree. The difference in benefits is entirely a result of the local character of the important processes. In order to investigate this hypothesis we simulated a forest with dispersed reproduction instead of local reproduction of the insects. The amount of colonized trees will be the same as in the previous simulation, but instead of reproduction into the $5 \times 5$ tree neighbourhood, new insects will be distributed randomly over the whole forest. The effect of this new reproduction scheme can be seen in Fig. 13. Mutants can survive in this system, but their abundance relative to wild-type late trees remains approximately the same throughout the simulation.

\section{Discussion}

All three models provide evidence about the effect of sequential predation on the competition between two prey species. The original coexistence of both prey species becomes destabilized when their common predator is present. Depending on initial conditions or model parameters the system will attain one of the two possible asymmetric equilibria with one prey species dominant. Furthermore, we have shown that due to the asymmetry in the two predator-prey relations there is an indirect positive effect of predator density on the late prey species. This asymmetry was first assumed in order to implement the sequentiality of the system in the DDS model. Results of the two other model formalisms not only verify this assumption, but indicate that this asymmetry might be stronger than previously expected. The results of the individual-oriented model in particular show that the presence of foliage-feeding insects gives a definite competitive advantage to the late tree. Due to this effect it might be a good strategy for the late species to be "attractive" to the predator. In the context of plant chemistry, this means that it would be a good strategy to invest in nutrients instead of toxins. This counterintuitive result can be understood if one realizes that such an adaptation can only be beneficial for a plant species if the direct disadvantage is compensated in some way by an indirect advantage. This is the case in a system of sequential predation: the first and principal victim of an increased number of predators will be the early prey species. The late prey species will also suffer more from increased predation pressure but this negative effect will be compensated by improved competition interaction with the early species. Therefore, if there are more predators, the late prey species will be more successful in its competition with the early prey species.

This phenomenon has strong similarities with the phenomenon of spiteful behaviour as described in the context of animal behaviour. An animal behaves spitefully when it harms itself in order to harm another individual more (Hamilton, 1970). It has been argued that spite may evolve only if it is expressed between individuals of less than average relatedness (Hamilton, 1970; Trivers, 1985). Hurst (1991) recently described the evolution of cytoplasmic incompatibility as another system in which spite can evolve. With the CA model (Section 6) we showed that a rare mutant with "spiteful" characteristics is indeed able to invade provided that the principal ecological processes are localized.

The basic asymmetry in our ecological system, which leads to a higher predation pressure upon the early prey, is closely related to the fact that there is only one generation of insects within each season. For species with a faster generation turnover (aphids), the asymmetric impact on the sequentially available prey species may be reversed (higher damage to the late prey).

We think that the main properties of this simple system of two prey species and one predator species will also be present in a multi-prey-multi-predator system. Thus, the demonstrated effect of sequential predation on the competition between prey species, the effect of spatial structure on population dynamics and the possibility for "spiteful" mutants to evolve in a spatial context can be expected to occur in other, more complex, ecosystems. However, since a sequential predator is able to change the outcome of the competition between prey species from coexistence to 
competitive exclusion, as demonstrated in this study, multi-prey systems may not be stable if common, sequential predators exist.

The effect of spatial structure of an ecosystem on both the ecological and evolutionary dynamics has been demonstrated in various parts of this model study. In Section 5.1 it is shown how the population dynamics of the larvae structures their own environment, which results in a "firewall" of defoliated trees around an island of late trees. This spatial structuring causes characteristic oscillatory dynamics, as can be seen clearly in Figs 3 and 4. We believe that this feedback loop between population dynamics and spatial structuring is an important factor for our understanding of ecosystems (see e.g. Hogeweg \& Hesper, 1990). In Section 5.2 we extend our study along these lines by using spatial patterns with a different scale of patchiness (size of regions dominated by one tree species) as initial configurations. Again the spatial structure appears to be an important factor influencing the population dynamics. The total length of the borderline between all areas occupied by early and late trees determines the speed of the competition process. A simulation with dispersed insect reproduction leads to a different outcome: extinction of larvae. The above three results clearly demonstrate the effect of spatial structure and localization of interactions on population dynamics on an ecological time scale. In Section 6.2 it is shown that localization of population dynamic interactions can have evolutionary consequences. The successful invasion by mutant trees of the late species can be explained by the fact that their effect remains localized. This results in a relatively higher share of the benefits for these mutant trees. We conclude that our study shows an alternative setting for the evolution of spiteful behaviour: a system with spatial structure.

The use of different model formalisms enables us to look at the system in different ways and highlight different aspects of the problem. The effect of sequential predation on the equilibria of the system can be investigated relatively simply with the DDS model. Bifurcation analysis using different parameters showed a clear difference in the effect of increased predation on the two prey species. Interpretation of these results is relatively easy given the simplicity of the model. A weakness of this model is of course the averaging over both space and time which is necessary to define the model.

The individual-oriented (Hobo) model as well as the cellular automaton are characterized by their explicit use of space, which appears to be an important aspect of this system. Both model formalisms also enable us to unravel the different processes in time and deal with processes on different time scales. A clear distinction has been made between yearly processes such as insect and tree reproduction and processes that occur within one season such as insect migration and feeding. In the CA model this distinction between processes on different time scales has been made in a qualitative way. In the Hobo model each process operates on its own appropriate scale.

The Hobo model generates quite a number of falsifiable hypotheses. Even though it is evident that the tree dynamics is too fast for oaks, it is nevertheless interesting to compare the simulation results to those obtained by Futuyma \& Wasserman (1980). In particular, all our simulations converged to the ultimate exclusion of early trees, so we never attained forest with sustained dominance of early trees, whereas Futuyma \& Wasserman (1980) observed both types of forest. Because most of the algorithms and parameters used in the Hobo model have a direct biological interpretation, it should be possible to find out the reason for this discrepancy. One possible explanation is suggested by the CA model (spatial gradient of external conditions). Also, the Hobo model can be easily modified or extended, given enough computer power (which seems to be the major limiting factor for this kind of modelling).

The CA model uses far fewer computer resources, yet preserves many of the important features of the Hobo model. Therefore, it can be useful for doing simulation experiments on a longer - evolutionarytime scale.

Each of the modelling formalisms thus has some advantages. However, we feel the greatest advantage is to be gained by using all three together and switching them iteratively. Then the results of one model formalism can provide a search image which can be pursued by means of another formalism.

We are grateful to Dr. Josef Jaroš and Dr. Jan Š. Lepš for discussing details of lepidopteran life cycles and forest dynamics, respectively. L. Lhotka is supported by CAS grant \#607112 and NWO Priority Programme for Non-Linear Systems. Finally, we thank Nick Savill for critical reading of the manuscript and Miss S. M. McNab for linguistic advice.

\section{REFERENCES}

Begon, M., Harper, J. L. \& Townsend, C. R. (1986). Ecology: Individuals, Populations and Communities. Oxford: Blackwell Scientific Publications.

De Boer, R. J., Van der Laan, J. D. \& Hogeweg, P. (1993). Randomness and pattern scale in the immune network: a cellular automaton approach. In: Thinking about Biology. SFI Studies in the Sciences of Complexity, Lecture Notes Vol. III (Stein, W. D. \& Varela, F. J. eds) Redwood City, CA: Addison-Wesley, pp. $231-252$. 
Dixon, A. F. G. (1985). Aphid Ecology. Glasgow: Blackie.

FutuYMa, D. J. \& WASSERMAN, S. S. (1980). Resource concentration and herbivory in oak forests. Science 210, 920-922.

GARDNER, M. (1970). Mathematical games: the fantastic combinations of John Conway's new solitaire game 'Life'. Sci. Am. $223(4), 120-123$.

Hamilton, W. D. (1970). Selfish and spiteful behaviour in an evolutionary model. Nature. Lond. 228, 1218-1220.

Hogeweg, P. (1988a). MIRROR beyond MIRROR, Puddles of LIFE. In: Artificial Life. SFI Studies in the Science of Complexity (Langton, E., ed) pp. 297-316. Redwood City, CA: AddisonWesley.

Hogeweg, P. (1988b). Cellular automata as a paradigm for ecological modelling. Appl. math. Comput. 27, 81-100.

HogeweG, P. (1989). Local T-T and T-B interactions: a cellular automaton approach. Immunol. Lett. 22, 113-122.

Hogeweg, P. \& Hesper, B. (1979). Heterarchical, selfstructuring simulation systems: concepts and applications in biology. In: Methodology in Systems Modelling and Simulation (Zeigler, B. P., Elzas, M. S., Klir, G. J., Ören, T. I. eds) pp. 221-232. Amsterdam: North-Holland.

Hogeweg, P. \& Hesper, B. (1990). Crowns crowding: an individual-oriented model of the Ancanthaster phenomenon. In: Ancanthaster and the Coral Reef: A Theoretical Perspective. (Bradbury, R. H., ed) Berlin-Heidelberg: Springer. pp. 169-188.

Holt, R. D. (1977). Predation, apparent competition, and the structure of prey communities. Theor. Popul. Biol. 12, 197-229.

Holt, R. D. (1984). Spatial heterogeneity, indirect interactions and the coexistence of prey species. Am. Nat. 124, 377-406.

HuRST, L. D. (1991). The evolution of cytoplasmatic incompatibility or when spite can be successful. J. theor. Biol. 148, 269-277.

Judson, O. P. (1994). The rise of the individual-based model in ecology. Trends. Ecol. Evol. 9, 9-14.

Lнотка, L. (1994). Implementation of individual-oriented models in aquatic ecology. Ecol. Model. 74, 47-62.

Mitter, C., Futuyma, D. J., Schneider, J. C. \& Hare, J. D. (1979). Genetic variation and host plant relations in a parthenogenetic moth. Evolution 33, 777-790.

ParcPlace Systems, Inc. (1992). Objectworks/Smalltalk, Version 4.1.

Tilman, D. (1982). Resource Competition and Community Structure. Princeton, NJ: Princeton University Press.

Toffoli, T. \& Margolus, N. (1987). Cellular Automata Machines. A New Environment for Modeling. Cambridge, MA: MIT Press.

Trivers, R. (1985). Social Evolution. Menlo Park, CA: The Benjamin/Cummings Publishing Company.

VAN DeR LaAn, J. D. \& Hogeweg, P. (1992). Waves of crown-of-thorns starfish outbreaks - where do they come from? Coral Reefs 11, 207-213.

VILLA, F. (1992). New computer architectures as tools for ecological thought. Trends Ecol. Evol. 7, 179-183.

Wolfram, S. (1984) Universality and complexity in cellular automata. Physica D 10, 1-35.

YodzIs, P. (1989). Introduction to Theoretical Ecology. New York: Harper and Row.

\section{APPENDIX A}

\section{Derivation of the DDS Model}

Our model is based on a simple Lotka-Volterra predator-prey model, with self-limitation for prey and a linear response curve for the predator-prey interaction. There is interspecific competition between the two prey species. So, if both prey species were simultaneously available the model would look like:

$$
\begin{aligned}
& X_{t+1}=X_{t}+a_{1} X_{t}-b_{1} X_{t}\left(X_{t}+g Y_{t}\right)-c_{1} X_{t} Z_{t} \\
& Y_{t+1}=Y_{t}+a_{2} Y_{t}-b_{2} Y_{t}\left(Y_{t}+g X_{t}\right)-c_{2} Y_{t} Z_{t} \\
& Z_{t+1}=Z_{t}-d Z_{t}+e_{2} c_{2} Y_{t} Z_{t}+e_{1} c_{1} X_{t} Z_{t} .
\end{aligned}
$$

Here, $X_{t}, Y_{t}$ and $Z_{t}$ are population densities of the two prey species and the predator, respectively, at time $t$

In order to introduce sequentiality into the predator-prey interactions we now decompose the processes within one season. With respect to the predator dynamics we distinguish four important moments within one season:

- $t_{0}$ is the beginning of the season.

- $t_{1}$ is the moment when the predators switch from early prey to late prey.

- $t_{2}$ is the moment, at the end of a season, at which predators start reproduction.

- $t_{3}:=t_{0}+1$ is the end of a season ( $=$ beginning of the next season).

In the intervals between these four points of the season the following processes occur:

From $t_{0}$ to $t_{1}$ : predators feed on early prey. The consumption per unit predator density is $c_{1} X$. Survival during this period is assumed to be completely dependent on the amount of early prey consumed. So the number of predators entering the second part of the season is

$$
Z\left(t_{1}\right)=Z\left(t_{0}\right) c_{1} X s_{1} .
$$

Since we interpret the term $s_{1} c_{1} X$ as a survival rate, it should be smaller than 1 for all feasible values of $X$ : $s_{1} c_{1} X_{\max } \leqslant 1$, with $X_{\max }=a_{1} / b_{1}$ (carrying capacity of $X$ )

From $t_{1}$ to $t_{2}$ : predators feed on late prey. The consumption per unit predator density is $c_{2} Y$. Survival during this second half of the season is assumed to be largely dependent on the amount of late prey consumed, but we also assume that a small fraction can survive independently of late prey:

$$
Z\left(t_{2}\right)=Z\left(t_{1}\right) c_{2} Y s_{2}+Z\left(t_{1}\right) s_{3}
$$

with $s_{2} c_{2} Y_{\max }+s_{3} \leqslant 1$.

From $t_{2}$ to $t_{3}$ : reproduction of the predators. With respect to reproduction we distinguish between predators which have been feeding on both early and late prey, and predators which have been feeding only on early prey (during the first half of the season):

$$
Z\left(t_{3}\right)=e_{2}\left(Z\left(t_{1}\right) c_{2} Y s_{2}\right)+e_{1}\left(Z\left(t_{1}\right) s_{3}\right) .
$$

Combination of the previous three equations gives:

$$
\begin{aligned}
Z\left(t_{3}\right) & =e_{2} Z\left(t_{1}\right) c_{2} Y s_{2}+e_{1} Z\left(t_{1}\right) s_{3} \\
& =e_{2} s_{1} s_{2} c_{1} c_{2} Z\left(t_{0}\right) X Y+e_{1} s_{1} s_{3} c_{1} Z\left(t_{0}\right) X .
\end{aligned}
$$


The decrease in prey density due to predation then becomes $c_{1} X Z\left(t_{0}\right)$ for early prey, and $c_{2} Y Z\left(t_{1}\right)=$ $s_{1} c_{2} c_{1} X Y Z\left(t_{0}\right)$ for late prey.

Using the above assumptions we modify the eqns (A.1) in the following way:

$$
\begin{aligned}
& X_{t+1}=X_{t}+a_{1} X_{t}-b_{1} X_{t}\left(X_{t}+g Y_{t}\right)-c_{1} X_{t} Z_{t} \\
& Y_{t+1}=Y_{t}+a_{2} Y_{t}-b_{2} Y_{t}\left(Y_{t}+g X_{t}\right)-s_{1} c_{2} c_{1} Y_{t} Z_{t} X_{t} \\
& Z_{t+1}=Z_{t}-d Z_{t}+e_{2} s_{1} s_{2} c_{1} c_{2} Y_{t} Z_{t} X_{t}+e_{1} s_{1} s_{3} c_{1} X_{t} Z_{t} .
\end{aligned}
$$

\begin{tabular}{|c|c|}
\hline$a_{1}, a_{2}$ & $\begin{array}{l}\text { natural rate of increase of prey } X \text { and } Y \text {, } \\
\text { respectively. }\end{array}$ \\
\hline$b_{1}, b_{2}$ & $\begin{array}{l}\text { competition coefficient of prey } X \text { and } \\
\text { respectively. }\end{array}$ \\
\hline$g:$ & $\begin{array}{l}\text { ratio between interspecific and intraspecific } \\
\text { competition. }\end{array}$ \\
\hline$c_{1}, c_{2}$ & $\begin{array}{l}\text { coefficient of functional response of prey } \\
\text { and } Y \text {, respectively. }\end{array}$ \\
\hline$s_{1}, s_{2}:$ & $\begin{array}{l}\text { survival rate of the predators when feeding } \\
\text { on prey } X \text { and } Y \text {, respectively. }\end{array}$ \\
\hline$s_{3}:$ & $\begin{array}{l}\text { survival rate of the predators who do not } \\
\text { feed during the second half of the season. }\end{array}$ \\
\hline$e_{1}:$ & $\begin{array}{l}\text { reproduction rate of the predators } \\
\text { have been feeding on prey } X \text { only. }\end{array}$ \\
\hline$e_{2}:$ & $\begin{array}{l}\text { reproduction rate of the } \\
\text { have been feeding on both }\end{array}$ \\
\hline
\end{tabular}

Definition of the parameters is as follows:

We used the following default parameter setting:

$$
\begin{gathered}
a_{1}=a_{2}=1 ; b_{1}=b_{2}=0.005 ; g=0.75 ; d=1 ; \\
c_{1}=0.05 ; c_{2}=0.15 ; \\
s_{1}=0.1 ; s_{2}=0.03 ; s_{3}=0.01 ; \\
e_{1}=0.01 ; e_{2}=10 .
\end{gathered}
$$

To simplify the model we lump some parameters in the following way:

$$
\begin{gathered}
c_{2}^{\prime}=s_{1} c_{2} c_{1}=0.00075 ; \\
e_{1}^{\prime}=e_{1} s_{1} s_{3}=0.00005 ; \\
e_{2}^{\prime}=e_{2} s_{2}=0.3 ;
\end{gathered}
$$

This gives the simplified model, where we again dropped the primes of the three lumped parameters

$$
\begin{aligned}
& X_{t+1}=X_{t}+a_{1} X_{t}-b_{1} X_{t}\left(X_{t}+g Y_{t}\right)-c_{1} X_{t} Z_{t} \\
& Y_{t+1}=Y_{t}+a_{2} Y_{t}-b_{2} Y_{t}\left(Y_{t}+g X_{t}\right)-c_{2} Y_{t} Z_{t} X_{t} \\
& Z_{t+1}=Z_{t}-d Z_{t}+e_{2} c_{2} Y_{t} Z_{t} X_{t}+e_{1} c_{1} X_{t} Z_{t} .
\end{aligned}
$$

\section{APPENDIX B}

\section{Implementation of the Hobo Model}

In this appendix we describe the standard version of the Hobo model. The parameters, functions and algorithms described here are used in all simulations unless otherwise specified.

The simulated forest is formed by a rectangular grid of patches. Every patch contains normally exactly one tree; exceptionally a patch can also be empty.

For both types of trees we distinguish 10 age classes. A new tree that takes over an empty patch is assigned the age $a:=1$. For all surviving trees this parameter is increased by 1 at the end of each season until $a=10$ is reached. From the age of 10 the trees are considered to be mature and they do not grow any more.

Every patch stores values of six parameters: $t$-tree species inhabiting the patch $(1=$ early, $2=$ late $)$, $f$-foliage (number of leaves), $a$-age, $g$-eggs (see below) and two values $h_{1}$ and $h_{2}$ representing the concept of a seedling bank. Both $h_{1}$ and $h_{2}$ are assigned zero values at the beginning of the simulation. Near the end of each season, a certain number of new seedlings of early and late trees are allocated to the patch, as described below. These numbers of new seedlings are added to $h_{1}$ and $h_{2}$, respectively. Then $h_{1}$ and $h_{2}$ are multiplied by a seedling survival rate-we used 0.3 throughout the simulations. The resulting values are used as $h_{1}$ and $h_{2}$ in the next season. In this way, the seedling bank keeps an account of the outcomes of previous years, with a minimum overhead of additional data structures.

Duration of a season was set for convenience at 100 simulation time units. The seasonal dynamics of trees consist of the following events (all times are given relative to the start of a particular season, i.e. on modulo 100);

(i) Budbreak. Budbreak of the early trees marks the beginning of each season, i.e. occurs at time $\beta_{1}=0$ days. Late trees break bud at $\beta_{2}=10$ days. After breaking bud the trees get fresh foliage. The initial number of leaves for a tree $p$ of species $i, i=1,2$, as a function of its age $a(p)$ is

$$
f_{0}(p):=f\left(p, \beta_{i}\right):=1400 \cdot a(p)+1000 .
$$

(ii) Reproduction. At time $\sigma=40$, i.e. after the larvae pupate, both tree species produce seeds which are locally dispersed and eventually make a contribution to the seedling banks of the corresponding patches. The number of viable seedlings produced by a tree $p$ depends on the 
species, age $a(p)$ and actual foliage $f(p, \sigma)$ of the tree $p$ :

$$
s(p):=\frac{a(p) f(p, \sigma) \theta(p)}{100} .
$$

The coefficient $\theta(p)$ depends on the species of tree $p$ : it is 1.1 for early trees and 1 for late trees. Increased production of seedlings for early trees is due to the fact that they start photosynthetic assimilation earlier.

Of the above mentioned number of seedlings, $20 \%$ are supposed to stay in the patch of the parent tree and the eight immediate neighbours get $10 \%$ each.

(iii) Forest update. Each season is closed at time $\omega$ (we set arbitrarily $\omega:=50$ ). At this moment, some of the trees die and are immediately replaced by new young trees. The probability of inter-seasonal survival again depends on the species of tree $p$, via the coefficient $\theta(p)$ :

$$
P_{s}(p):=\left(1-\frac{P_{a}(p)}{\theta(p)}\right) \theta(p) P_{f}(p) .
$$

Age-dependent mortality has the probability $P_{a}(p):=0.05 / a(p)$, i.e. young trees are more vulnerable. Survival is also less likely for defoliated trees, as expressed in the term

$$
P_{f}(p):=\min \left\{1, \frac{f(p, \omega)+3000}{f_{0}(p)}\right\},
$$

where $f_{0}(p)$ is the initial (full) foliage given by (B.1). Note that even completely defoliated trees have a non-zero probability of survival.

An early tree replaces a dead tree in a patch $p$ with probability

$$
P_{e}(p):=h_{1}(p) /\left(h_{1}(p)+h_{2}(p)\right)
$$

for the early species and symmetrically for late tree. These probabilities reflect the species' relative abundance in the seedling bank.

Another seasonally varying property of trees is important for the dynamics of larvae - the leaf palatability $q(p)$. Leaf palatability depends on the leaf maturation $\tau(p)$ (number of days since budbreak of the tree $p$ ) in the following way:

$$
q_{i}(\tau):=\left\{\begin{array}{lc}
1, & \text { if } \tau \leqslant 8 \\
(28-\tau) / 20, & \text { otherwise }
\end{array}\right.
$$

Larvae are implemented as Hobo populations, see Lhotka (1994), which can be thought of as sets of equivalent individuals. In the present model this equivalence relation is based on weight. Weight $w(l)$ of a larval population $l$ increases continuously in the range 0-22. A weight class (or population in the terminology of the Hobo system) contains all larvae in a given patch whose weight gives the same value when rounded to the nearest integer. Thus, more weight classes may coexist in a single patch.

The development of larvae in the model is divided into four simulated instars. At times of 8, 16, 24 and 32 days after hatching the larvae face "deadlines": populations with weights of less than $3,8,13$ and 18, respectively, are killed. On the other hand, whenever a population reaches the weight of 22 , it immediately finishes the larval stage. The idea of instars was used mainly for improving the efficiency of computer simulation since populations with bad performance are eradicated in earlier stages of the simulation run.

The life cycle of larvae consists of alternating feeding and migration. For a larval population $l$ on a tree $p$, the decision about what to do next is made as follows: If the tree $p$ is highly defoliated, or its leaves unpalatable, the entire population $l$ splits up and moves to neighbouring patches. Otherwise, each individual of all larval populations on $p$ is allocated a fair share of the available foliage. If this share is not enough to satiate the population $l$, part of $l$ migrates, in proportion to the ratio of available and desired amount of food. The remaining larvae then eat their share of leaves and grow accordingly. In precise terms, the criteria are as follows:

(i) If foliage $f(p)$ is less than 100 , or leaf palatability $q(p)$ is less than 0.05 , the entire population migrates (the algorithm for strong migration is used, see below).

(ii) Otherwise, the amount of food available for each individual larva of the population is determined thus:

$$
e(p, l):=\min \left\{\frac{f(p)}{N(p)}, E(l)\right\},
$$

where $f(p)$ is the actual foliage and $N(p)$ is the total number of larvae on the tree $p$, that is, the sum of population sizes of all populations on the tree $p$, including $l$. The term $E(l)$ represents food demand of an individual larva of the population $l$ which grows exponentially with the larval weight: $E(l):=3 \exp (0.08 w(l))$.

(iii) Weight of population $l$ is increased by $e(p, l) q(p) / 7$, and population $l$ then waits for $e(p, l)$ days. Duration of feeding and digestion is equal to $d(p, l):=\mathrm{e}(p, l) / E(l)$, i.e. the 
population $l$ is inactive for 1 day if its food demand is completely satisfied.

(iv) Foliage of tree $p$ is decreased by $e(p, l) n(l)$ where $n(l)$ is the size of population $l$.

(v) If the relative satiation $d(p, l)$ is less than 0.1 , the entire population $l$ leaves the tree $p$ - the algorithm for strong migration is used again. Otherwise, only a part of population $l$ migrates, using another way of migration, called weak migration (see below). The fraction of larvae that migrate is $1-0.9 d(p, l) q(p)$.

Two migration algorithms have been mentioned: Weak migration is assumed to take place via overlapping branches of neighbouring trees. Therefore, the migrants move, with equal likelihood, to one of four direct neighbours in horizontal and vertical directions.

Strong migration, on the other hand, means that the larvae use all possible ways to reach neighbouring trees, including dangerous ones like descending to the ground and looking for another tree. Hence we assume in this case that $20 \%$ of the population do not survive this action. The remainder are distributed over all eight surrounding patches. Each of the horizontal and vertical neighbours gets, on average, $15 \%$ of the population, whereas the diagonal neighbours get $10 \%$ each, on average. Populations that migrate in this way can resume activities (on other trees) after 0.3 days (migration period).

Care has been taken not to make dispersal of larvae too isotropic. On the other hand, this frequent operation has been made as computer-efficient as possible. So for small populations (typically of size less than 100) a random sample of the corresponding multinomial distribution is generated, where the percentages attached to the neighbouring patches are used as probabilities. Larger populations are divided exactly according to the percentages.

The model-independent mortality rate of larvae was set at $0.05 \mathrm{day}^{-1}$. For large populations this amount is simply discounted from their population size, while for smaller populations probabilistic mortality is applied on the individual-by-individual basis.

In this model we do not follow the life history of the insect after it completes the larval stage. All we need to know is the distribution of eggs at the beginning of the next season. To this end, we assumed that the sex ratio in the adult population is 1:1. Each larva of population $l$ which later becomes female yields the following number of viable eggs for the next season: $g(l):=25 \cdot\left(w_{f}(l)+18\right)+50$, where $w_{f}(l)$ is the weight of $l$ at the end of the larval development. Hence, for the minimum weight of $18, g(l)$ is 50 , and for maximum $w_{f}(l)=22$ the number of eggs is 150 .

Even more important than the number of eggs is the way in which these eggs are distributed over the space. In the standard variant females are assumed to be wingless. An individual female is assumed to lay eggs in the patch where it completed larval development with probability 0.2 , and in each of the eight immediately neighbouring patches with probability 0.1 .

Boundary conditions are specific for different spatial processes: The boundary is open with respect to the dispersion of seeds and eggs and strong migration, whereas it is closed for weak migration.

The Smalltalk source code of the Hobo model can be obtained from anonymous FTP server baloun.entu.cas.cz, directory/pub/hobo/seqpred.

\section{APPENDIX C}

\section{Implementation of the CA Model}

The cellular automaton consists of $240 \times 120$ cells. The variables of a cell are tree and insect. A cell can be either empty or occupied with respect to each of these two variables. We distinguish three different tree types: early species, late species and a mutant of late species.

Periodic boundary conditions have been used in the simulation of Section 6.1 in the main text; we therefore assume a forest on a torus. In the forest with a gradient (Section 6.2 and 6.3) periodic boundary conditions have been used for the vertical direction (top and bottom boundary), whereas open boundaries are used for the left and right boundary.

\section{TREE DYNAMICS}

- Local (density-dependent) reproduction: if a cell is empty, it chooses one of the eight surrounding neighbours at random. The empty cell will change its state to the state of the chosen neighbour. If this neighbour happens to be empty as well, the cell will remain empty.

- Global mortality $\left(M_{g}\right)$ : a tree can die each year with probability $M_{g}$. In the simulations of Section 6.1 this parameter has value $M_{g}=0.03$ for all tree species. In Section 6.2 and 6.3 the global mortality of early species is dependent on the position of a tree on a (left-to-right) gradient. On the far left side of the forest $M_{g}(1)=0.02$, whereas on the far right side of the forest $M_{g}(1)=0.04$. The global mortality for trees of late species and mutant species is the same all along the gradient: $M_{g}(2)=M_{g}(m)=0.03$.

- Local (density-dependent) mortality $\left(M_{l}\right)$ : if a cell is occupied by a tree of a certain species, its 
probability of becoming empty is proportional to the number of surrounding trees of the same species. Every cell chooses one of its eight surrounding cells at random. If the chosen neighbouring cell is occupied by the same species, the probability of dying is $5 \%$ $\left(M_{l}=0.05\right)$.

\section{INSECT DYNAMICS}

- Local reproduction $\left(P_{r}\right)$ : At the end of a season insects will reproduce and spread to a subset of the trees in the neighbourhood. Insects which have been feeding on a tree of late species during the second part of the season will reproduce and spread to $50 \%$ (randomly chosen) of the $5 \times 5$ surrounding neighbourhood $\left(P_{r}(2)=0.5\right)$. Insects on a tree of mutant species will reproduce and spread to $95 \%$ of this neighbourhood $\left(P_{r}(m)=0.95\right)$.

- Influx (I): At the beginning of every season a small fraction $(I=0.001)$ of the trees will receive insects. We added this property to the CA-model in order to guarantee the presence of insects during the whole simulation.

- Migration: insects migrate by diffusion. We here use the cellular automaton diffusion model of Toffoli \& Margolus (1987) with particle conservation. The migration is simulated by performing five consecutive diffusion steps. This implies that insects can move no further than a maximum of five trees away.

\section{INTERACTION BETWEEN INSECTS AND TREES}

There is no interaction between insects and trees during the migration of the insects.

- Survival: During the first part of the season insects can only live on trees of early species. So, if they are in a cell without a tree or with a tree of late species (or its mutant), they will die. During the second part of the season this situation changes: insects in empty cells or on early species will die, insects on late species (or its mutant) will survive.
- Damage $\left(M_{i}\right)$ : If a tree is occupied by an insect its probability of dying increases. In the first part of a season, when insects live only on early species, the probability that a cell will become empty is $35 \%\left(M_{i}(1)=0.35\right)$. In the second part of the season, when insects live only on late species (or its mutant), this probability is higher $M_{i}(2)=M_{i}(m)=1.0$. In this way we take into account the fact that insects grow larger during the first part of the season and, as a result, will eat more during the second part of the season.

\section{TIMING OF ACTIVITIES}

We model the different processes within one year subsequently:

(1) local (density-dependent) mortality of trees

(2) global mortality of trees

(3) global influx of insects

(4) mortality of insects which are not on early trees

(5) damage to trees (early)

(6) migration of insects

(7) mortality of insects which are not on late trees (or mutant)

(8) damage to trees (late and mutant)

(9) reproduction of trees

(10) reproduction of insects.

\section{SIMULATION}

A simulation starts with an initialization of the forest with $50 \%$ early and $50 \%$ late, randomly distributed. The model then runs for 300 time steps without insects, in order to produce a mixed forest. The situation after these 300 years is then taken as the initial distribution of trees for the simulation with insects. Mutant trees are introduced by changing $5 \%$ of the trees of the late species to the mutant species. 